\title{
Finite-Time Blow-Up and Local Existence for Chemotaxis System with a General Memory Term
}

\author{
WU Yingdong*, WU Shaohua, CHENG Xin and LIU Lei \\ School of Mathematics and Statistics, Wuhan University, Wuhan 430072, China.
}

Received 16 August 2020; Accepted 31 October 2020

\begin{abstract}
In this paper, we discuss the local existence of weak solutions for a parabolic system modelling chemotaxis with memory term, and we show the finite-time blowup and chemotactic collapse for this system. The main methods we used are the fixed point theorem and the semigroup theory.
\end{abstract}

AMS Subject Classifications: 35A01, 35A02, 35K51

\section{Chinese Library Classifications: O175.4}

Key Words: parabolic system; chemotaxis; memory term; fixed point theorem; blow-up.

\section{Introduction}

In this paper, we consider the following model:

$$
\begin{cases}u_{t}=\nabla(\nabla u-\chi u \nabla v)+\int_{0}^{t} f[u(\cdot, \tau)] \mathrm{d} \tau & \text { in } \Omega \times(0, T), \\ v_{t}=\Delta v-v+u & \text { in } \Omega \times(0, T), \\ u(\cdot, 0)=u_{0} & \text { in } \Omega \times\{0\}, \\ v(\cdot, 0)=v_{0} & \text { in } \Omega \times\{0\}, \\ \frac{\partial u}{\partial \vec{n}}=\frac{\partial v}{\partial \vec{n}}=0 & \text { on } \partial \Omega \times(0, T),\end{cases}
$$

*Corresponding author. Email addresses: 15755508002@163.com (Y. D. Wu), s.h.wu@163.com (S. H. Wu), chengxin021@whu.edu.cn (X. Cheng), liulei95@whu.edu.cn (L. Liu) 
where $\Omega \subset \mathbb{R}^{N}$, a bounded open domain with smooth boundary $\partial \Omega, \vec{n}$ is the unit outer normal on $\partial \Omega$ and $\chi$ is a nonnegative constant. And $f$ is a continuous linear function and it satisfies the condition: $\left\|f\left(x_{1}\right)-f\left(x_{2}\right)\right\| \leq L\left\|x_{1}-x_{2}\right\|$, where $L$ is a positive constant.

Our model is initiated by the PKS model which is a mathematical model of biological phenomena. And this model for chemosensitive movement has been developed by Patlak, Keller and Segel [1].

$$
\left\{\begin{array}{l}
u_{t}=\nabla(\nabla u-\chi(v) u \nabla v), \\
\varepsilon v_{t}=\Delta v+g(u, v),
\end{array}\right.
$$

where $u$ represents the population density and $v$ denotes the density of the external stimulus, $\chi$ is the sensitive coefficient, the time constant $\varepsilon(0 \leq \varepsilon \leq 1)$ indicates that the spatial spread of the organisms $u$ and the signal $v$ are on different time scales. The case $\varepsilon=0$ corresponds to a quasi-steady state assumption for the signal distribution.

Since the PKS model is designed to describe the behavior of bacteria and bacteria aggregates, the question arises whether or not this model is able to show aggregation. Plenty of theoretical research uncovered exact conditions for aggregations and for blow up (see, e.g., Childress and Percus [2,3], Jäger and Luckhaus [4], Nagai [5], Gajewski et al. [6], Senba [7], Rasde and Ziti [8], Herrera and Velasquez [9], Othmer and Stevens [10] or Levine and Sleeman [11]).

Global existence below these thresholds has been proven using a Lyapunov functional in Gajewski, et al. [6], Nagai, et al. [12] and Biler [13]. Besides, a number of theoretical research found exact conditions for aggregations and other properties [14-16]. Free boundary problems for the chemotaxis model are considered [17-20].

Our study of (1.1) is also motivated by the following problem for the heat equation with a general time integral boundary condition [21]:

$$
\begin{cases}u_{t}=\Delta u & x \in \Omega, t>0, \\ \frac{\partial u}{\partial \vec{n}}=\int_{0}^{t} f[u(x, s)] \mathrm{d} s & x \in \partial \Omega, t>0, \\ u(x, 0)=u_{0}(x) & x \in \bar{\Omega},\end{cases}
$$

where $\Omega$ is a bounded domain in $\mathbb{R}^{N}$ with boundary $\partial \Omega \subset C^{1+\mu}(0<\mu<1), \vec{n}$ is the outward normal, and $u_{0}(x)$ is a nonnegative function such that

$$
\frac{\partial u_{0}}{\partial \vec{n}}=0 \quad \text { for } x \in \partial \Omega,
$$

$f$ is a nondecreasing function with $f \in C^{1}(0, \infty)$ and $f(0)>0$.

Considering the nonlinear time integral condition governing flux through the boundary, the model (1.1) involves a continuous time delay which is often referred to as a memory condition in the literature. This memory term can perfectly describe the movement 
of population density or the movement of single particles. Especially, the movement behavior of most species is guided by external signals: insects orient towards light sources, the smell of a sexual partner makes it favorable to choose a certain direction.

Models with memory terms present in the boundary flux have been formulated in many applied sciences. For example, in [22], a linear memory boundary condition is introduced for the study of thermodynamics. It takes into account the hereditary effects on the boundary as those studied in $[23,24]$. Similar hereditary boundary conditions have been employed in models of time-dependent electromagnetic fields at dissipative boundaries [25].

From the mathematical point of view, it is significant to study the local existence of weak solution for chemotaxis system with memory terms and the finite-time blow-up for this system. In our previous work, we have done something for this [26].

\section{Some basic lemmas}

Choose a constant $\sigma$ which satisfies

$$
1<\sigma<2
$$

and

$$
N<2 \sigma<N+2 .
$$

It is easy to check that (2.1) and (2.2) can be simultaneously satisfied in the case of $1 \leq N \leq 3$. We define

$$
\begin{aligned}
& X_{u}=C\left(\left[0, t_{0}\right], H^{\sigma}(\Omega) \cap\left\{\frac{\partial u}{\partial \vec{n}}=0 \text { on } \partial \Omega\right\}\right), \\
& X_{v}=C\left(\left[0, t_{0}\right], H^{2}(\Omega) \cap\left\{\frac{\partial v}{\partial \vec{n}}=0 \text { on } \partial \Omega\right\}\right), \\
& X=H^{\sigma}(\Omega) \cap\left\{\frac{\partial u}{\partial \vec{n}}=0 \text { on } \partial \Omega\right\} .
\end{aligned}
$$

Here, $u(x, t) \in C\left(\left[0, t_{0}\right], H^{\sigma}(\Omega) \cap\left\{\frac{\partial u}{\partial \vec{n}}=0\right.\right.$ on $\left.\left.\partial \Omega\right\}\right)$ means that $u(x, t) \in H^{\sigma}(\Omega) \cap\left\{\frac{\partial u}{\partial \vec{n}}=\right.$ 0 on $\partial \Omega\}$ for each $t \in\left[0, t_{0}\right]$ and $\|u(\cdot, t)\|_{H^{\sigma}} \in C\left(\left[0, t_{0}\right]\right)$. In the Sections 2 and 3 , inessential constants will be denoted by the same letter $c$, even if they may vary from line to line.

Lemma 2.1. Let $p(z)$ be a holomorphic semigroup on a Banach space $Y$, with generator $A$. Then

$$
t>0, f \in Y \Rightarrow p(t) f \in D(A),
$$

and

$$
\|A p(t) f\|_{Y} \leq \frac{c}{t}\|f\|_{Y,} \text { for } 0<t \leq 1
$$


Proof. The proof can be found in [27, Proposition 7.2].

If $\Omega$ is a bounded open domain with smooth boundary, on which the Neumann boundary condition is placed, then we know that $e^{t \Delta}$ defines a holomorphic semigroup on the Hilbert space $L^{2}(\Omega)$. So by Lemma 2.1, we have that

$$
f \in L^{2}(\Omega) \Rightarrow\left\|e^{t \Delta} f\right\|_{H^{2}(\Omega)} \leq \frac{c}{t}\|f\|_{L^{2}(\Omega)}
$$

where

$$
D(\Delta)=\left\{u \in H^{2}(\Omega), x \frac{\partial u}{\partial \vec{n}}=0 \text { on } \partial \Omega\right\} .
$$

Applying interpolation to (2.3) yields

$$
\left\|e^{t \Delta} f\right\|_{H^{\sigma}(\Omega)} \leq c t^{-\frac{\sigma}{2}}\|f\|_{L^{2}(\Omega)} \quad \text { for } 1<\sigma<2,0<t \leq 1 .
$$

Lemma 2.2. We assume that $u \in X$, a Banach space of functions, and that there is another Banach space $Y$ such that the following four conditions hold:

$$
\begin{aligned}
& e^{t \Delta}: X \rightarrow X \text { is a strongly continuous semigroup, for } t \geq 0, \\
& \Psi: X \rightarrow Y \text { is Lipschitz, uniformly on bounded sets, } \\
& e^{t \Delta}: Y \rightarrow X, \quad \text { for } t>0,
\end{aligned}
$$

and, for some $\gamma<1$,

$$
\left\|e^{t \Delta}\right\|_{\mathcal{L}(Y, X)} \leq C t^{-\gamma}, \quad \text { for } t \in(0,1] .
$$

Then we have a bound $\|\Psi(u(s))\|_{Y} \leq K_{1}$ and

$$
\left\|\int_{0}^{t} e^{(t-s) \Delta} \Psi(u(s)) \mathrm{d} s\right\|_{X} \leq C_{\gamma} t^{1-\gamma} K_{1} .
$$

Proof. The proof can be found in [27].

Dividing system (1.1) into two parts:

$$
\begin{cases}u_{t}=\nabla(\nabla u-\chi u \nabla v)+\int_{0}^{t} f[u(\cdot, \tau)] \mathrm{d} \tau & \text { in } \Omega \times(0, T), \\ u(\cdot, 0)=u_{0} & \text { in } \Omega \times\{0\}, \\ \frac{\partial u}{\partial \vec{n}}=0 & \text { on } \partial \Omega \times(0, T),\end{cases}
$$


and

$$
\begin{cases}v_{t}=\Delta v-v+u & \text { in } \Omega \times(0, T), \\ v(\cdot, 0)=v_{0} & \text { in } \Omega \times\{0\}, \\ \frac{\partial v}{\partial \vec{n}}=0 & \text { on } \partial \Omega \times(0, T),\end{cases}
$$

then we have the following lemmas.

Lemma 2.3. For $u \in X_{u}, v_{0} \in H^{2}(\Omega) \cap\left\{\frac{\partial v}{\partial \vec{n}}=0\right.$ on $\left.\partial \Omega\right\}, t_{0}>0$ small enough, problem (2.6) has a unique solution $v \in X_{v}$, and $v$ satisfies

$$
\|v(\cdot, t)\|_{X_{v}} \leq c\left\|v_{0}\right\|_{H^{2}}+c t_{0}^{1-\frac{\sigma}{2}} \sup _{0 \leq t \leq t_{0}}\|u(\cdot, t)\|_{L^{2}}, \quad 0 \leq t \leq t_{0},
$$

where $c$ is a constant which is independent of $T$.

Proof. It is obvious that Eq. (2.6) has a solution and the solution is unique. So what we need to proof is (2.7). Let $T(t)=e^{t \Delta}$, where $D(\Delta)=H^{2}(\Omega) \cap\left\{\frac{\partial v}{\partial \vec{n}}=0\right.$ on $\left.\partial \Omega\right\}$, then

$$
v(t)=T(t) v_{0}-\int_{0}^{t} T(t-s) v(s) \mathrm{d} s+\int_{0}^{t} T(t-s) u(s) \mathrm{d} s .
$$

By (2.3), Lemma 2.2, we calculate

$$
\begin{aligned}
\|v(\cdot, t)\|_{H^{2}} & \leq c\left\|v_{0}\right\|_{H^{2}}+c t_{0}^{1-\frac{\sigma}{2}} \sup _{0 \leq t \leq t_{0}}\|v(\cdot, t)\|_{L^{2}}+c t_{0}^{1-\frac{\sigma}{2}} \sup _{0 \leq t \leq t_{0}}\|u(\cdot, t)\|_{L^{2}} \\
& \leq c\left\|v_{0}\right\|_{H^{2}}+c t_{0}^{1-\frac{\sigma}{2}} \sup _{0 \leq t \leq t_{0}}\|v(\cdot, t)\|_{H^{2}}+c t_{0}^{1-\frac{\sigma}{2}} \sup _{0 \leq t \leq t_{0}}\|u(\cdot, t)\|_{L^{2}}, \quad 0 \leq t \leq t_{0} .
\end{aligned}
$$

Thus for small enough $t_{0},(2.7)$ holds.

Lemma 2.4. For each $u_{0} \in H^{\sigma}(\Omega)$ and $v \in X_{v}, \sigma$ and $N$ satisfy (2.1) and (2.2). The problem (2.5) has a unique solution $u \in c\left(\left[0, t_{0}\right], H^{\sigma}(\Omega)\right)$, and the solution can be written as

$$
\begin{aligned}
u(t)=T(t) & u_{0}-\chi \int_{0}^{t} T(t-s) \nabla u \nabla v \mathrm{~d} s-\chi \int_{0}^{t} T(t-s) u \Delta v \mathrm{~d} s \\
& +\int_{0}^{t} T(t-s) \int_{0}^{s} f(u) \mathrm{d} \tau \mathrm{d} s .
\end{aligned}
$$

Proof. We consider the following problem first

$$
\begin{cases}u_{t}=\Delta u-\chi \nabla(\tilde{u} \nabla v)+\int_{0}^{t} f[\tilde{u}(\cdot, \tau)] \mathrm{d} \tau & \text { in } \Omega \times(0, T), \\ u(\cdot, 0)=u_{0} & \text { in } \Omega \times\{0\}, \\ \frac{\partial u}{\partial \vec{n}}=0 & \text { on } \partial \Omega \times(0, T),\end{cases}
$$


where $\tilde{u} \in X_{u}$ is fixed. Using the semigroup notation $T(t)=e^{t \Delta}$, we can write the solution of (2.9) as

$$
\begin{gathered}
u(t)=T(t) u_{0}-\chi \int_{0}^{t} T(t-s) \nabla v \nabla \tilde{u} \mathrm{~d} s-\chi \int_{0}^{t} T(t-s) \tilde{u} \Delta v \mathrm{~d} s \\
+\int_{0}^{t} T(t-s) \int_{0}^{s} f[\tilde{u}(\cdot, \tau)] \mathrm{d} \tau \mathrm{d} s .
\end{gathered}
$$

Define a mapping

$$
\begin{aligned}
& G_{1}: X_{u} \rightarrow X_{u}, \\
& G_{1}(\tilde{u})=u,
\end{aligned}
$$

where $u$ is the corresponding solution of (2.9).

Then we claim that for $t_{0}$ small enough, $G_{1}$ is a contract mapping. In fact, let $\tilde{u}_{1}, \tilde{u}_{2} \in X_{u}$, we have

$$
\begin{aligned}
G_{1}\left(\tilde{u}_{1}\right)-G_{1}\left(\tilde{u}_{2}\right)=-\chi & \int_{0}^{t} T(t-s) \nabla\left(\left(\tilde{u}_{1}-\tilde{u}_{2}\right) \nabla v\right) \mathrm{d} s \\
& +\int_{0}^{t} T(t-s) \int_{0}^{s}\left(f\left[\tilde{u}_{1}(\cdot, \tau)\right]-f\left[\tilde{u}_{2}(\cdot, \tau)\right]\right) \mathrm{d} \tau \mathrm{d} s .
\end{aligned}
$$

By Sobolev imbedding theorems, we have

$$
\begin{aligned}
& H^{1}(\Omega) \hookrightarrow L^{\infty}(\Omega) \quad \text { for } N=1, \\
& H^{1}(\Omega) \hookrightarrow L^{q}(\Omega), 1<q<\infty \quad \text { for } N=2, \\
& H^{1}(\Omega) \hookrightarrow L^{\frac{2 N}{N-2}}(\Omega) \quad \text { for } N=3 \text {. }
\end{aligned}
$$

If $N=1$,

$$
\begin{aligned}
& \quad\left\|\chi \nabla\left(\tilde{u}_{1}-\tilde{u}_{2}\right) \nabla v\right\|_{L^{2}} \leq \chi\left\|\nabla\left(\tilde{u}_{1}-\tilde{u}_{2}\right)\right\|_{L^{2}}\|\nabla v\|_{L^{\infty}} \\
& \leq c\left\|\tilde{u}_{1}-\tilde{u}_{2}\right\|_{H^{1}}\|\nabla v\|_{H^{1}} \leq c\left\|\tilde{u}_{1}-\tilde{u}_{2}\right\|_{H^{\sigma}}\|v\|_{H^{2}} .
\end{aligned}
$$

If $N=2,3$, according to (2.1) and (2.2), we obtain that

$$
H^{1}(\Omega) \hookrightarrow L^{\frac{N}{\sigma-1}}(\Omega), \quad H^{\sigma-1}(\Omega) \hookrightarrow L^{\frac{2 N}{N-2(\sigma-1)}}(\Omega) .
$$

We know that $\nabla\left(\tilde{u}_{1}-\tilde{u}_{2}\right) \in H^{\sigma-1}$ and $\nabla v \in H^{1}$, then $\left|\nabla\left(\tilde{u}_{1}-\tilde{u}_{2}\right)\right|^{2} \in L^{\frac{N}{N-2(\sigma-1)}}(\Omega)$ and $|\nabla v|^{2} \in$ $L^{\frac{N}{2(\sigma-1)}}(\Omega)$. Hence, Hölder's inequality yields

$$
\left\|\left|\nabla\left(\tilde{u}_{1}-\tilde{u}_{2}\right)\right|^{2} \cdot|\nabla v|^{2}\right\|_{L^{1}} \leq\left\|\left|\nabla\left(\tilde{u}_{1}-\tilde{u}_{2}\right)\right|^{2}\right\|_{L^{\frac{N}{N-2(\sigma-1)}}}\left\||\nabla v|^{2}\right\|_{L^{\frac{N}{2(\sigma-1)}}},
$$


which implies

$$
\left\|\chi \nabla\left(\tilde{u}_{1}-\tilde{u}_{2}\right) \nabla v\right\|_{L^{2}} \leq \chi\left\|\nabla\left(\tilde{u}_{1}-\tilde{u}_{2}\right)\right\|_{L^{\frac{2 N}{N-2(\sigma-1)}}}\|\nabla v\|_{L^{\frac{N}{\sigma-1}}} \leq c\left\|\tilde{u}_{1}-\tilde{u}_{2}\right\|_{H^{\sigma}}\|v\|_{H^{2}} .
$$

Hence for $N=1,2,3$, we have

$$
\left\|\chi \nabla\left(\tilde{u}_{1}-\tilde{u}_{2}\right) \nabla v\right\|_{L^{2}} \leq c\left\|\tilde{u}_{1}-\tilde{u}_{2}\right\|_{H^{\sigma}}\|v\|_{H^{2}} .
$$

Similarly, we have

$$
\left\|\chi\left(\tilde{u}_{1}-\tilde{u}_{2}\right) \Delta v\right\|_{L^{2}} \leq c\left\|\tilde{u}_{1}-\tilde{u}_{2}\right\|_{H^{\sigma}}\|v\|_{H^{2}} .
$$

So for the first term on the right side of (2.10), $N=1,2,3$, by (2.11) and (2.12), we have

$$
\begin{aligned}
& \left\|\int_{0}^{t} T(t-s) \nabla\left(\left(\tilde{u}_{1}-\tilde{u}_{2}\right) \nabla v\right) \mathrm{d} s\right\|_{H^{\sigma}} \\
\leq & c t_{0}^{1-\frac{\sigma}{2}} \sup _{0 \leq s \leq t}\left\|\nabla\left(\left(\tilde{u}_{1}-\tilde{u}_{2}\right) \nabla v\right)\right\|_{L^{2}} \\
\leq & c t_{0}^{1-\frac{\sigma}{2}} \sup _{0 \leq s \leq t}\left\{\left\|\nabla\left(\tilde{u}_{1}-\tilde{u}_{2}\right) \nabla v\right\|_{L^{2}}+\left\|\left(\tilde{u}_{1}-\tilde{u}_{2}\right) \Delta v\right\|_{L^{2}}\right\} \\
\leq & c t_{0}^{1-\frac{\sigma}{2}} \sup _{0 \leq s \leq t}\left\|\tilde{u}_{1}-\tilde{u}_{2}\right\|_{H^{\sigma}}\|v\|_{H^{2}} .
\end{aligned}
$$

For the second term on the right side of (2.10), we have

$$
\begin{aligned}
& \left\|\int_{0}^{t} T(t-s) \int_{0}^{s}\left(f\left[\tilde{u}_{1}(\cdot, \tau)\right]-f\left[\tilde{u}_{2}(\cdot, \tau)\right]\right) \mathrm{d} \tau \mathrm{d} s\right\|_{H^{\sigma}} \\
\leq & \int_{0}^{t}\left\|T(t-s) \int_{0}^{s}\left(f\left[\tilde{u}_{1}(\cdot, \tau)\right]-f\left[\tilde{u}_{2}(\cdot, \tau)\right]\right) \mathrm{d} \tau\right\|_{H^{\sigma}} \mathrm{d} s \\
\leq & \int_{0}^{t} c(t-s)^{-\frac{\sigma}{2}}\left\|\int_{0}^{s}\left(f\left[\tilde{u}_{1}(\cdot, \tau)\right]-f\left[\tilde{u}_{2}(\cdot, \tau)\right]\right) \mathrm{d} \tau\right\|_{L^{2}} \mathrm{~d} s \\
\leq & c t_{0}^{1-\frac{\sigma}{2}} \sup _{0 \leq s \leq t}\left\|\int_{0}^{s}\left(\tilde{u}_{1}-\tilde{u}_{2}\right) \mathrm{d} \tau\right\|_{L^{2}} \leq c t_{0}^{1-\frac{\sigma}{2}} \sup _{0 \leq s \leq t} \int_{0}^{s}\left\|\left(\tilde{u}_{1}-\tilde{u}_{2}\right)\right\|_{H^{\sigma}} \mathrm{d} \tau \\
\leq & c t_{0}^{1-\frac{\sigma}{2}} \sup _{0 \leq s \leq t 0 \leq \tau \leq s} \sup _{0 \leq \tilde{u}_{1}-\tilde{u}_{2}\left\|_{H^{\sigma}} \leq c t_{0}^{1-\frac{\sigma}{2}} \sup _{0 \leq \tau \leq t}\right\| \tilde{u}_{1}-\tilde{u}_{2} \|_{H^{\sigma}} .}
\end{aligned}
$$

So we have

$$
\left\|G_{1}\left(\tilde{u}_{1}\right)-G_{1}\left(\tilde{u}_{2}\right)\right\|_{X_{u}} \leq c t_{0}^{1-\frac{\sigma}{2}}\left\|\tilde{u}_{1}-\tilde{u}_{2}\right\|_{X_{u}}\|v\|_{X_{v}}+c t_{0}^{1-\frac{\sigma}{2}}\left\|\tilde{u}_{1}-\tilde{u}_{2}\right\|_{X_{u}},
$$


which implies for $t_{0}>0$ small enough, $G_{1}$ is contract. By Banach fixed point theorem, there exists a unique fixed point $\tilde{u}$ such that $G_{1}(\tilde{u})=\tilde{u}$. Then we have the local solution of the problem (2.5):

$$
\begin{gathered}
u(t)=T(t) u_{0}-\chi \int_{0}^{t} T(t-s) \nabla u \nabla v \mathrm{~d} s-\chi \int_{0}^{t} T(t-s) u \Delta v \mathrm{~d} s \\
+\int_{0}^{t} T(t-s) \int_{0}^{s} f(u) \mathrm{d} \tau \mathrm{d} s .
\end{gathered}
$$

This completes the proof of the lemma.

Lemma 2.5. Assume $\sigma, N$ as given by (2.1) and (2.2). For solution $u \in X_{u}$ of (2.5), we have

$$
\|u\|_{X_{u}} \leq c\left\|u_{0}\right\|_{H^{\sigma}}+c t_{0}^{1-\frac{\sigma}{2}}\|v\|_{X_{v}}\|u\|_{X_{u}}, 0 \leq t \leq t_{0} .
$$

Proof. By Lemma 2.3, (2.5) has a unique solution, and the solution can be written as

$$
\begin{aligned}
u(t)=T(t) & u_{0}-\chi \int_{0}^{t} T(t-s) \nabla u \nabla v \mathrm{~d} s-\chi \int_{0}^{t} T(t-s) u \Delta v \mathrm{~d} s \\
& +\int_{0}^{t} T(t-s) \int_{0}^{s} f(u) \mathrm{d} \tau \mathrm{d} s .
\end{aligned}
$$

Next we prove estimate (2.13). By (2.4) we have

$$
\left\|\int_{0}^{t} T(t-s) \nabla u \nabla v \mathrm{~d} s\right\|_{H^{\sigma}} \leq c t_{0}^{1-\frac{\sigma}{2}} \sup _{0 \leq s \leq t}\|\nabla u(\cdot, s) \nabla v(\cdot, s)\|_{L^{2}} .
$$

By Sobolev imbedding theorem, $H^{1}(\Omega) \hookrightarrow L^{\infty}(\Omega)$ for $N=1$, we have

$$
\|\nabla u \nabla v\|_{L^{2}} \leq\|\nabla u\|_{L^{2}}\|\nabla v\|_{L^{\infty}} \leq c\|u\|_{H^{1}}\|v\|_{H^{2}} \leq c\|u\|_{H^{\sigma}}\|v\|_{H^{2}} .
$$

For $N=2,3$, we have

$$
\begin{aligned}
& \quad\|\nabla u \nabla v\|_{L^{2}} \leq\|\nabla u\|_{L^{\frac{2 N}{N-2(\sigma-1)}}}\|\nabla v\|_{L^{\frac{N}{\sigma-1}}} \leq c\|\nabla u\|_{L^{\frac{2 N}{N-2(\sigma-1)}}}\|\nabla v\|_{H^{1}} \\
& \leq c\|\nabla u\|_{H^{\sigma-1}}\|v\|_{H^{2}} \leq c\|u\|_{H^{\sigma}}\|v\|_{H^{2}} .
\end{aligned}
$$

So we obtain that, for $0 \leq t \leq t_{0}$,

$$
\begin{gathered}
\left\|\int_{0}^{t} T(t-s) \nabla u \nabla v \mathrm{~d} s\right\|_{H^{\sigma}} \leq c t^{1-\frac{\sigma}{2}} \sup _{0 \leq s \leq t}\|\nabla u \nabla v\|_{L^{2}} \\
\leq c t^{1-\frac{\sigma}{2}} \sup _{0 \leq s \leq t}\|u\|_{H^{\sigma}}\|v\|_{H^{2}} \leq c t_{0}^{1-\frac{\sigma}{2}}\|u\|_{X_{u}}\|v\|_{X_{v}} .
\end{gathered}
$$


Meanwhile, we deduce

$$
\begin{aligned}
&\left\|\int_{0}^{t} T(t-s) u \Delta v \mathrm{~d} s\right\|_{H^{\sigma}} \leq c t^{1-\frac{\sigma}{2}} \sup _{0 \leq s \leq t}\|u \Delta v\|_{L^{2}} \\
& \leq c t_{0}^{1-\frac{\sigma}{2}} \sup _{0 \leq s \leq t_{0}}\|u\|_{L^{\infty}}\|\Delta v\|_{L^{2}} \leq c t_{0}^{1-\frac{\sigma}{2}} \sup _{0 \leq s \leq t_{0}}\|u\|_{H^{\sigma}} \sup _{0 \leq s \leq t_{0}}\|v\|_{H^{2}} \leq c t_{0}^{1-\frac{\sigma}{2}}\|u\|_{X_{u}}\|v\|_{X_{v}},
\end{aligned}
$$

and

$$
\begin{aligned}
& \left\|\int_{0}^{t} T(t-s) \int_{0}^{s} f[u(\cdot, \tau)] \mathrm{d} \tau \mathrm{d} s\right\|_{H^{\sigma}} \leq \int_{0}^{t}\left\|T(t-s) \int_{0}^{s} f(u) \mathrm{d} \tau\right\|_{H^{\sigma}} \mathrm{d} s \\
\leq & \int_{0}^{t} c(t-s)^{-\frac{\sigma}{2}}\left\|\int_{0}^{s} f(u) \mathrm{d} \tau\right\|_{L^{2}} \mathrm{~d} s \leq c t_{0}^{1-\frac{\sigma}{2}} \sup _{0 \leq s \leq t}\left\|\int_{0}^{s} f(u) \mathrm{d} \tau\right\|_{L^{2}} \\
\leq & c t_{0}^{1-\frac{\sigma}{2}} \sup _{0 \leq s \leq t} \int_{0}^{s}\|u\|_{H^{\sigma}} \mathrm{d} \tau \leq c t_{0}^{1-\frac{\sigma}{2}} \sup _{0 \leq s \leq t 0 \leq \tau \leq s} \sup _{0 \leq s}\|u\|_{H^{\sigma}} \leq c t_{0}^{1-\frac{\sigma}{2}} \sup _{0 \leq \tau \leq t_{0}}\|u\|_{H^{\sigma}} .
\end{aligned}
$$

Hence we declare that

$$
\begin{aligned}
&\|u(t)\|_{H^{\sigma}} \leq\left\|T(t) u_{0}\right\|_{H^{\sigma}}+\chi \| \int_{0}^{t} T(t-s) \nabla u \nabla v \mathrm{~d} s \\
&+\chi\left\|\int_{0}^{t} T(t-s) u \Delta v \mathrm{~d} s\right\|_{H^{\sigma}}+\left\|\int_{0}^{t} T(t-s) \int_{0}^{s} u \mathrm{~d} \tau \mathrm{d} s\right\|_{H^{\sigma}} \\
& \leq c\left\|u_{0}\right\|_{H^{\sigma}}+c t_{0}^{1-\frac{\sigma}{2}}\|u\|_{X_{u}}\|v\|_{X_{v}}+c t_{0}^{1-\frac{\sigma}{2}}\|u\|_{X_{u}}, \quad 0 \leq t \leq t_{0},
\end{aligned}
$$

which implies for $t_{0}$ small enough

$$
\|u(t)\|_{X_{u}} \leq c\left\|u_{0}\right\|_{H^{\sigma}}+c t_{0}^{1-\frac{\sigma}{2}}\|u\|_{X_{u}}\|v\|_{X_{v}} .
$$

Thus, Lemma 2.5 is proved.

\section{Local existence of solution}

In this section, we establish the local solution of system (1.1).

Theorem 3.1. Under conditions (2.1) and (2.2), for each initial data $u_{0} \in X, v_{0} \in H^{2}(\Omega) \cap\left\{\frac{\partial v}{\partial \vec{n}}=\right.$ 0 on $\partial \Omega\}$, problem (1.1) has a unique solution $(u, v) \in X_{u} \times X_{v}$ for some $t_{0}>0$.

Proof. Consider $g \in X_{u}$ and $g(x, 0)=u_{0}(x)$ and let $v=v(g)$ denotes the corresponding solution of the equation

$$
\begin{cases}v_{t}=\Delta v-v+g & \text { in } \Omega \times\left(0, t_{0}\right), \\ v(\cdot, 0)=v_{0} & \text { in } \Omega \times\{0\}, \\ \frac{\partial v}{\partial \vec{n}}=0 & \text { on } \partial \Omega \times\left(0, t_{0}\right) .\end{cases}
$$


By Lemma 2.2, we have $v \in X_{v}$ and

$$
\|v(\cdot, t)\|_{X_{v}} \leq c\left\|v_{0}\right\|_{H^{2}}+c t_{0}^{1-\frac{\sigma}{2}} \sup _{0 \leq t \leq t_{0}}\|g(\cdot, t)\|_{L^{2}}, \quad t \in\left(0, t_{0}\right) .
$$

For the solution $v$ of (3.1), define $u=u(v(g))$ to be the corresponding solution of

$$
\begin{cases}u_{t}=\nabla(\nabla u-\chi u \nabla v)+\int_{0}^{t} f[u(\cdot, \tau)] \mathrm{d} \tau & \text { in } \Omega \times\left(0, t_{0}\right), \\ u(x, 0)=u_{0}(x) & \text { in } \Omega \times\{0\}, \\ \frac{\partial u}{\partial \vec{n}}=0 & \text { on } \partial \Omega \times\left(0, t_{0}\right) .\end{cases}
$$

Define a mapping

$$
G_{2} g=u(v(g)) .
$$

Then Lemma 2.3 shows that $G_{2}: X_{u} \rightarrow X_{u}$. Take $M=2 c\left\|u_{0}\right\|_{H^{\sigma}}$ and a ball

$$
B_{M}=\left\{g \in X_{u} \mid:\|g(\cdot, t)\|_{H^{\sigma}} \leq M, g(x, 0)=u_{0}(x), 0 \leq t \leq t_{0}\right\},
$$

where the constant $c$ is given by (2.13). Then we conclude from (2.13) and (3.2) that

$$
\begin{aligned}
\left\|G_{2} g\right\|_{X_{u}} & \leq c\left\|u_{0}\right\|_{H^{\sigma}}+c t_{0}^{1-\frac{\sigma}{2}}\|v\|_{X_{v}}\left\|G_{2} g\right\|_{X_{u}} \\
& \leq c\left\|u_{0}\right\|_{H^{\sigma}}+c t_{0}^{1-\frac{\sigma}{2}}\left(c\left\|v_{0}\right\|_{H^{2}}+c t_{0}^{1-\frac{\sigma}{2}} \sup _{0 \leq t \leq t_{0}}\|g(\cdot, t)\|_{L^{2}}\right)\left\|G_{2} g\right\|_{X_{u}} .
\end{aligned}
$$

If $g \in B_{M},\|g\|_{L^{2}} \leq c\|g\|_{H^{\sigma}} \leq c M$, then for $t_{0}>0$ small enough $\left\|G_{2} g\right\|_{X_{u}} \leq 2 c\left\|u_{0}\right\|_{H^{\sigma}}$. So for $t_{0}>0$ small enough, $G_{2}$ maps $B_{M}$ into $B_{M}$.

Next we demonstrate that for $t_{0}$ small enough, $G_{2}$ is a contract mapping. In fact, let $g_{1}, g_{2} \in B_{M} \subset X_{u}$ and $v_{1}, v_{2}$ denote the corresponding solutions of (3.1). Then

$$
\begin{aligned}
& G_{2} g_{1}-G_{2} g_{2}=u_{1}-u_{2} \\
& =-\chi \int_{0}^{t} T(t-s)\left(u_{1} \Delta v_{1}-u_{2} \Delta v_{2}\right) \mathrm{d} s-\chi \int_{0}^{t} T(t-s)\left(\nabla u_{1} \nabla v_{1}-\nabla u_{2} \nabla v_{2}\right) \mathrm{d} s \\
& \quad+\int_{0}^{t} T(t-s) \int_{0}^{s} f\left(u_{1}\right)-f\left(u_{2}\right) \mathrm{d} \tau \mathrm{d} s .
\end{aligned}
$$

For the first term on the right side of (3.4),

$$
\begin{aligned}
& \left\|\int_{0}^{t} T(t-s)\left(u_{1} \Delta v_{1}-u_{2} \Delta v_{2}\right) \mathrm{d} s\right\|_{H^{\sigma}} \\
\leq & \left\|\int_{0}^{t} T(t-s) u_{1}\left(\Delta v_{1}-\Delta v_{2}\right) \mathrm{d} s\right\|_{H^{\sigma}}+\left\|\int_{0}^{t} T(t-s)\left(u_{1}-u_{2}\right) \Delta v_{2} \mathrm{~d} s\right\|_{H^{\sigma}},
\end{aligned}
$$


where

$$
\begin{aligned}
& \left\|\int_{0}^{t} T(t-s) u_{1}\left(\Delta v_{1}-\Delta v_{2}\right) \mathrm{d} s\right\|_{H^{\sigma}} \leq c t_{0}^{1-\frac{\sigma}{2}} \sup _{0 \leq s \leq t_{0}}\left\|u_{1}\left(\Delta v_{1}-\Delta v_{2}\right)\right\|_{H^{\sigma}} \\
\leq & c t_{0}^{1-\frac{\sigma}{2}} \sup _{0 \leq s \leq t_{0}}\left\|u_{1}\right\|_{L^{\infty}}\left\|\Delta\left(v_{1}-v_{2}\right)\right\|_{L^{2}} \leq c M t_{0}^{1-\frac{\sigma}{2}} \sup _{0 \leq s \leq t_{0}}\left\|v_{1}-v_{2}\right\|_{H^{2}},
\end{aligned}
$$

and

$$
\begin{aligned}
&\left\|\int_{0}^{t} T(t-s)\left(u_{1}-u_{2}\right) \Delta v_{2} \mathrm{~d} s\right\|_{H^{\sigma}} \leq c t_{0}^{1-\frac{\sigma}{2}} \sup _{0 \leq s \leq t_{0}}\left\|\left(u_{1}-u_{2}\right) \Delta v_{2}\right\|_{L^{2}} \\
& \leq c t_{0}^{1-\frac{\sigma}{2}} \sup _{0 \leq s \leq t_{0}}\left\|v_{2}\right\|_{H^{2}}\left\|u_{1}-u_{2}\right\|_{L^{\infty}} \leq c t_{0}^{1-\frac{\sigma}{2}}\left\|v_{2}\right\|_{X_{v}}\left\|u_{1}-u_{2}\right\|_{X_{u}} .
\end{aligned}
$$

Therefore

$$
\begin{gathered}
\left\|\int_{0}^{t} T(t-s)\left(u_{1} \Delta v_{1}-u_{2} \Delta v_{2}\right) \mathrm{d} s\right\|_{H^{\sigma}} \\
\leq c t_{0}^{1-\frac{\sigma}{2}}\left\|v_{1}-v_{2}\right\|_{X_{v}}+c t_{0}^{1-\frac{\sigma}{2}}\left\|v_{2}\right\|_{X_{v}}\left\|u_{1}-u_{2}\right\|_{X_{u}},
\end{gathered}
$$

where $0 \leq t \leq t_{0}$. For the second term on the right side of (3.4), we have

$$
\begin{aligned}
& \left\|\int_{0}^{t} T(t-s)\left(\nabla u_{1} \nabla v_{1}-\nabla u_{2} \nabla v_{2}\right) \mathrm{d} s\right\|_{H^{\sigma}} \\
\leq & \left\|\int_{0}^{t} T(t-s)\left(\nabla u_{1} \nabla v_{1}-\nabla u_{2} \nabla v_{1}\right) \mathrm{d} s\right\|_{H^{\sigma}}+\left\|\int_{0}^{t} T(t-s)\left(\nabla u_{2} \nabla v_{1}-\nabla u_{2} \nabla v_{2}\right) \mathrm{d} s\right\|_{H^{\sigma}},
\end{aligned}
$$

where

$$
\begin{gathered}
\left\|\int_{0}^{t} T(t-s)\left(\nabla u_{1} \nabla v_{1}-\nabla u_{2} \nabla v_{1}\right) \mathrm{d} s\right\|_{H^{\sigma}} \\
\leq c t_{0}^{1-\frac{\sigma}{2}} \sup _{0 \leq t \leq t_{0}}\left\|\nabla v_{1} \nabla\left(u_{1}-u_{2}\right)\right\|_{L^{2}}, \quad 0 \leq t \leq t_{0} .
\end{gathered}
$$

As we have done in Lemma 2.3 and 2.4, we obtain that

$$
\left\|\int_{0}^{t} T(t-s)\left(\nabla u_{1} \nabla v_{1}-\nabla u_{2} \nabla v_{1}\right) \mathrm{d} s\right\|_{H^{\sigma}} \leq c t_{0}^{1-\frac{\sigma}{2}}\left\|v_{1}\right\|_{X_{v}}\left\|u_{1}-u_{2}\right\|_{X_{u}}, 0 \leq t \leq t_{0} .
$$

Similarly

$$
\left\|\int_{0}^{t} T(t-s)\left(\nabla u_{2} \nabla v_{1}-\nabla u_{2} \nabla v_{2}\right) \mathrm{d} s\right\|_{H^{\sigma}} \leq c t_{0}^{1-\frac{\sigma}{2}} \sup _{0 \leq t \leq t_{0}}\left\|\nabla u_{2} \nabla\left(v_{1}-v_{2}\right)\right\|_{L^{2}}
$$




$$
\leq c t_{0}^{1-\frac{\sigma}{2}}\left\|u_{2}\right\|_{X_{u}}\left\|v_{1}-v_{2}\right\|_{X_{v}} \leq c M t_{0}^{1-\frac{\sigma}{2}}\left\|v_{1}-v_{2}\right\|_{X_{v}}, \quad 0 \leq t \leq t_{0}
$$

Then

$$
\begin{gathered}
\left\|\int_{0}^{t} T(t-s)\left(\nabla u_{1} \nabla v_{1}-\nabla u_{2} \nabla v_{2}\right) \mathrm{d} s\right\|_{H^{\sigma}} \\
\leq c t_{0}^{1-\frac{\sigma}{2}}\left\|v_{1}\right\|_{X_{v}}\left\|u_{1}-u_{2}\right\|_{X_{u}}+c t_{0}^{1-\frac{\sigma}{2}}\left\|v_{1}-v_{2}\right\|_{X_{v}}, \quad 0 \leq t \leq t_{0} .
\end{gathered}
$$

For the last term on the right side of (3.4), we have

$$
\left\|\int_{0}^{t} T(t-s) \int_{0}^{s} f\left(u_{1}\right)-f\left(u_{2}\right) \mathrm{d} \tau \mathrm{d} s\right\|_{H^{\sigma}} \leq c t_{0}^{1-\frac{\sigma}{2}}\left\|u_{1}-u_{2}\right\|_{X_{u}} .
$$

Combining the estimates (3.5), (3.6), and (3.7), it follows that

$$
\begin{aligned}
& \quad\left\|G_{2} g_{1}-G_{2} g_{2}\right\|_{X_{u}} \\
& \leq c t_{0}^{1-\frac{\sigma}{2}}\left\|v_{1}-v_{2}\right\|_{X_{v}}+c t_{0}^{1-\frac{\sigma}{2}}\left\|v_{2}\right\|_{X_{v}}\left\|u_{1}-u_{2}\right\|_{X_{u}} \\
& \quad+c t_{0}^{1-\frac{\sigma}{2}}\left\|v_{1}\right\|_{X_{v}}\left\|u_{1}-u_{2}\right\|_{X_{u}}+c t_{0}^{1-\frac{\sigma}{2}}\left\|v_{1}-v_{2}\right\|_{X_{v}}+c t_{0}^{1-\frac{\sigma}{2}}\left\|u_{1}-u_{2}\right\|_{X_{u}},
\end{aligned}
$$

which implies

$$
\begin{aligned}
& \left\|G_{2} g_{1}-G_{2} g_{2}\right\|_{X_{u}} \\
\leq & 2 c t_{0}^{1-\frac{\sigma}{2}}\left\|v_{1}-v_{2}\right\|_{X_{v}}+c t_{0}^{1-\frac{\sigma}{2}}\left(\left\|v_{2}\right\|_{X_{v}}+\left\|v_{1}\right\|_{X_{v}}+1\right)\left\|G_{2} g_{1}-G_{2} g_{2}\right\|_{X_{u}} .
\end{aligned}
$$

Consider the following equation

$$
\begin{cases}\left(v_{1}-v_{2}\right)_{t}=\Delta\left(v_{1}-v_{2}\right)-\left(v_{1}-v_{2}\right)+\left(g_{1}-g_{2}\right) & \text { in } \Omega \times\left(0, t_{0}\right), \\ \left(v_{1}-v_{2}\right)(\cdot, 0)=0 & \text { in } \Omega \times\{0\}, \\ \frac{\partial\left(v_{1}-v_{2}\right)}{\partial \vec{n}}=0 & \text { on } \partial \Omega \times\left(0, t_{0}\right) .\end{cases}
$$

By (2.7), we obtain

$$
\left\|v_{1}-v_{2}\right\|_{X_{v}} \leq c t_{0}^{1-\frac{\sigma}{2}} \sup _{0 \leq t \leq t_{0}}\left\|g_{1}-g_{2}\right\|_{L^{2}} \leq c t_{0}^{1-\frac{\sigma}{2}} \sup _{0 \leq t \leq t_{0}}\left\|g_{1}-g_{2}\right\|_{H^{\sigma}}
$$

Moreover, we have

$$
\left\|v_{1}\right\|_{X_{v}} \leq c\left\|v_{0}\right\|_{H^{2}}+c t_{0}^{1-\frac{\sigma}{2}} \sup _{0 \leq t \leq t_{0}}\left\|g_{1}\right\|_{L^{2}} \leq c\left\|v_{0}\right\|_{H^{2}}+c t_{0}^{1-\frac{\sigma}{2}} M, \quad 0 \leq t \leq t_{0}
$$




$$
\left\|v_{2}\right\|_{X_{v}} \leq c\left\|v_{0}\right\|_{H^{2}}+c t_{0}^{1-\frac{\sigma}{2}} \sup _{0 \leq t \leq t_{0}}\left\|g_{2}\right\|_{L^{2}} \leq c\left\|v_{0}\right\|_{H^{2}}+c t_{0}^{1-\frac{\sigma}{2}} M, \quad 0 \leq t \leq t_{0} .
$$

Thus for $t_{0}>0$ small enough, $G_{2}$ is contract.

From the process above, we have proved that problem (1.1) has a solution $(u, v) \in$ $X_{u} \times X_{v}$ by Lemmas 2.2, 2.3 and 2.4. We derive the uniqueness by Banach fixed point theorem.

\section{Blow-up in finite time}

We then introduce an auxiliary function $F(u)$ defined by

$$
F(u)=\int_{0}^{u} f(\sigma) \mathrm{d} \sigma .
$$

And we suppose that $N=1$, then we have the following result.

Theorem 4.1. If $f(u)$ is a convex function on $[0, \infty)$, and $F(u)$ satisfies

$$
\int_{0}^{\infty} F^{-1 / 2}(u) \mathrm{d} u<\infty,
$$

then all nonnegative solutions of (1.1) blow up in finite time.

Proof. In the section, without causing any confusion, we may use $C_{i}(i=0,1,2 \ldots)$ to denote various positive constants.

$$
u_{t}=\nabla(\nabla u-\chi u \nabla v)+\int_{0}^{\tau} f[u(x, s)] \mathrm{d} s .
$$

Integrate both sides of the equation on $\Omega$,

$$
\frac{\mathrm{d}}{\mathrm{dt}} \int_{\Omega} u \mathrm{~d} x=\int_{\Omega}(\nabla(\nabla u-\chi u \nabla v)) \mathrm{d} x+\int_{\Omega} \int_{0}^{\tau} f[u(x, s)] \mathrm{d} s \mathrm{~d} x .
$$

By using $\frac{\partial u}{\partial \vec{n}}=\frac{\partial v}{\partial \vec{n}}=0$, we can get the following equality:

$$
\int_{\Omega} u \mathrm{~d} x-\int_{\Omega} u_{0} \mathrm{~d} x=\int_{\Omega} \int_{0}^{\tau} \int_{0}^{t} f[u(x, s)] \mathrm{d} s \mathrm{~d} x \mathrm{~d} t
$$

Set

$$
K(t)=\int_{\Omega} u(x, t) \mathrm{d} x \quad \text { for } \quad t>0 .
$$

By using Jensen's inequality, we find

$$
C_{1} \int_{0}^{t} \int_{0}^{\tau} f(K(s)) \mathrm{d} s \mathrm{~d} \tau \leq \int_{\Omega} \int_{0}^{\tau} \int_{0}^{t} f[u(x, s)] \mathrm{d} s \mathrm{~d} x \mathrm{~d} t .
$$


Then we can get that $K(t)$ satisfies

$$
K(t) \geq C_{2}+C_{1} \int_{0}^{t} \int_{0}^{\tau} f(K(s)) \mathrm{d} s \mathrm{~d} \tau \quad t>0 .
$$

Assume to the contrary that (1.1) has a global solution $u$. Then for any positive number $\mathrm{T}$, we have

$$
K(t) \geq C_{2}+C_{1} \int_{T}^{\tau} \int_{T}^{t} f(K(s)) \mathrm{d} s \mathrm{~d} \tau \quad \text { for } T \leq t \leq 2 T .
$$

Thus, by comparision, $K(t) \geq k(t)$ on $[T, 2 T]$, where

$$
k(t) \geq C_{2}+C_{1} \int_{T}^{\tau} \int_{T}^{t} f(k(s)) \mathrm{d} s \mathrm{~d} \tau \quad \text { for } \leq t \leq 2 T .
$$

Clearly, $k(t)$ satisfies

$$
\begin{aligned}
& k^{\prime \prime}(t)=C_{1} f(k(t)), \quad T<t<2 T, \\
& k(T)=C_{2}, k^{\prime}(T)=0 .
\end{aligned}
$$

Multiplying the equation in (4.2) by $k^{\prime}(t)$ and integrating from $T$ to $t$, we obtain

$$
k^{\prime}(t)=C_{3}|F(k(t))-F(k(T))|^{1 / 2} .
$$

Integration of this relation over $(\mathrm{T}, 2 \mathrm{~T})$ then leads to

$$
\begin{aligned}
C_{3} T & =\int_{k(T)}^{k(2 T)}[F(z)-F(k(T))]^{-1 / 2} \mathrm{~d} z \\
& \leq \int_{k(T)}^{c}[F(z)-F(k(T))]^{-1 / 2} \mathrm{~d} z+\int_{c}^{\infty}[F(z)-F(k(T))]^{-1 / 2} \mathrm{~d} z \\
& \leq\left[f\left(C_{2}\right)\right]^{-1 / 2} \int_{C_{2}}^{c}\left[z-C_{2}\right]^{-1 / 2} \mathrm{~d} z+\int_{c}^{\infty}[F(z)-F(z) / 2]^{-1 / 2} \mathrm{~d} z \\
& =2\left(c-C_{2}\right)^{1 / 2} f^{-1 / 2}\left(C_{2}\right)+\sqrt{2} \int_{c}^{\infty} F^{-1 / 2}(z) \mathrm{d} z,
\end{aligned}
$$

where $\mathrm{c}$ is a positive constant chosen so that $F(c)=2 F(k(T))=2 F\left(C_{2}\right)$. For sufficiently large $T$, inequality (4.3) yields a contraction to condition (4.1), which completes the proof.

\section{Ackhowledgement}

We would like to thank the referees and our advisor for carefully reading the manuscript and for their helpful suggestions. 


\section{References}

[1] Keller E. F., Segel L. A., Initiation of slime mold aggregation viewed as an instability. Journal of theoretical biology, 26(3) (1970) 399-415.

[2] Childress S. Chemotactic collapse in two dimensions. In Modelling of patterns in space and time, 61-66. Springer, 1984.

[3] Childress S., Percus J. K., Nonlinear aspects of chemotaxis. Mathematical Biosciences, 56(3-4) (1981) 217-237.

[4] Jäger W., Luckhaus S., On explosions of solutions to a system of partial differential equations modelling chemotaxis. Transactions of the american mathematical society, 329(2) (1992) 819-824.

[5] Nagai T., Blow-up of radially symmetric solutions to a chemotaxis system. Advances in Mathematical Sciences and Applications, 5 (1995) 581-601.

[6] Gajewski H., Zacharias K. and Gröger K., Global behaviour of a reaction-diffusion system modelling chemotaxis. Mathematische Nachrichten, 195(1) (1998) 77-114.

[7] Senba T., Blow-up of radially symmetric solutions to some systems of partial differential equations modelling chemotaxis. Advances in Mathematical Sciences and Applications, 7 (1997) 79-92.

[8] Rascle M., Ziti C., Finite time blow-up in some models of chemotaxis. Journal of mathematical biology, 33(4) (1995) 388-414.

[9] Herrero M. A., Velázquez J. J., Singularity patterns in a chemotaxis model. Mathematische Annalen, 306(1) (1996) 583-623.

[10] Stevens A., Othmer H. G., Aggregation, blowup, and collapse: the abc's of taxis in reinforced random walks. SIAM Journal on Applied Mathematics, 57(4) (1997) 1044-1081.

[11] Sleeman B. D., Levine H. A., A system of reaction diffusion equations arising in the theory of reinforced random walks. SIAM Journal on Applied Mathematics, 57(3) (1997) 683-730.

[12] Nagai T., Senba T. and Yoshida K., Application of the trudinger-moser inequality to a parabolic system of chemotaxis. Funkcialaj Ekvacioj, 40(3) (1997) 411-434.

[13] Biler P., Local and global solvability of some parabolic systems modelling chemotaxis. Advances in Mathematical Sciences and Applications, 8 (1998) 715-743.

[14] Lv W. B., Wang Q. Y., Global existence for a class of chemotaxis systems with signaldependent motility, indirect signal production and generalized logistic source. Zeitschrift für angewandte Mathematik und Physik, 71(2) (2020) 1-18.

[15] Lv W. B., Wang Q., A chemotaxis system with signal-dependent motility, indirect signal production and generalized logistic source: Global existence and asymptotic stabilization. Journal of Mathematical Analysis and Applications, (2020) page 124108.

[16] Chen $\mathrm{H}$. and $\mathrm{Wu} \mathrm{S}$. H., On existence of solutions for some hyperbolicparabolic-type chemotaxis systems. IMA Journal of Applied Mathematics, 2007.

[17] Chen H., Lv W. B. and Wu S. H., A free boundary problem for a class of parabolic type chemotaxis model. Kinetic and Related Models, 8(4) (2015) 667.

[18] Wu S. H., A free boundary problem for a chemotaxis system. Acta Mathematica Sinica. Chinese Series, 53 (2010) 515-524.

[19] Chen H., Lv. W. B. and Wu S. H., Solvability of a parabolic-hyperbolic type chemotaxis system in 1-dimensional domain. Acta Mathematica entia, 36 (005) (2016) 1285-1304.

[20] Chen H., Lv W. B. and Wu S. H., A free boundary problem for a class of parabolic-elliptic type chemotaxis model. Communications on Pure and Applied Analysis, 17(6) (2018) 2577-2592.

[21] Deng K., Dong Z. H., Blow-up for the heat equation with a general memory boundary 
condition. Communications on Pure and Applied Analysis, 11(5) (2012) 2147.

[22] Ciarletta M., A differential problem for heat equation with a boundary condition with memory. Applied Mathematics Letters, 10(1):95-101, 1997.

[23] Fabrizio M. and Morro A., Mathematical Problems in Linear Viscoelasticity. SIAM, 1992.

[24] Gurtin M. A., Pipkin A. C., A general theory of heat conduction with finite wave speeds. Archive for Rational Mechanics and Analysis, 31(2) (1968) 113-126.

[25] Fabrizio M., Morro A., A boundary condition with memory in electromagnetism. Archive for rational mechanics and analysis, 136(4)(1996) 359-381.

[26] Wu S. H., Liu L., Existence of solutions for a parabolic system modelling chemotaxis with memory term. Journal of Partial Differential Equations, 33(2) (2020) 158.

[27] Michael M. T., Taylor M., Partial differential equations. iii. Applied Mathematical Sciences, 117, 1999. 